\title{
New Existence of Solutions for Fractional Integro-Differential Equations with Nonseparated Boundary Conditions
}

\author{
Lahcen Ibnelazyz $\mathbb{D}^{D}$, Karim Guida $\mathbb{D}^{D}$, Khalid Hilal $\mathbb{D}$, and Said Melliani \\ Sultan Moulay Slimane University, BP 523, Beni Mellal 23000, Morocco \\ Correspondence should be addressed to Lahcen Ibnelazyz; ibnelazyzlahcen@gmail.com
}

Received 2 April 2021; Revised 7 May 2021; Accepted 11 June 2021; Published 24 June 2021

Academic Editor: Xinan Hao

Copyright (C) 2021 Lahcen Ibnelazyz et al. This is an open access article distributed under the Creative Commons Attribution License, which permits unrestricted use, distribution, and reproduction in any medium, provided the original work is properly cited.

Results reported in this article prove the existence and uniqueness of solutions for a class of nonlinear fractional integro-differential equations supplemented by nonseparated boundary value conditions. We consider a new norm to establish the existence of solution via Krasnoselskii fixed point theorem; however, the uniqueness results are obtained by applying the contraction mapping principle. Some examples are provided to illustrate the results.

\section{Introduction}

Fractional differential equations have been an important tool to describe many problems and processes in different fields of science. In fact, fractional models are more realistic than the classical models. Fractional differential equations appear in many fields such as physics, economics, image processing, blood flow phenomena, aerodynamics, and so on. For more details about fractional differential equations and their applications, we provide the following references [1-13].

Recently, fractional integro-differential equations were investigated by many researchers in different problems, and a lot of papers were published in this matter (see, for example, [14-16]).
Furthermore, many boundary conditions were considered for the fractional-order integro-differential equations; some of these conditions are the classical, periodic, antiperiodic, nonlocal, multipoint, and the integral boundary conditions (for details, see [17-22]).

On the other hand, many papers have considered the nonseparated boundary conditions as they are a very important class of boundary value conditions (we refer the readers to [23-28]).

Motivated by the above discussion, in this paper, we establish the existence and uniqueness of solutions for a class of fractional integro-differential equations with nonseparated boundary value conditions as follows:

$$
\left\{\begin{array}{l}
\left.{ }^{c} D^{\alpha}\left({ }^{c} D^{\beta}\right) x(t)=f(t, x(t), \phi x(t), \psi x(t))\right), \quad t \in[0,1], \\
x(0)=\lambda_{1} x(1), \\
x^{\prime}(0)=\lambda_{2} x^{\prime}(1), \\
{ }^{c} D^{\beta} x(0)=\lambda_{3}{ }^{c} D^{\beta} x(1), \\
{ }^{c} D^{\beta+1} x(0)=\lambda_{4}{ }^{c} D^{\beta+1} x(1),
\end{array}\right.
$$


where $1<\alpha \leq 2,1<\beta \leq 2, \lambda_{1}, \lambda_{2}, \lambda_{3}, \lambda_{4} \in \mathbb{R}-\{0,1\},{ }^{c} D^{\beta},{ }^{c} D^{\alpha}$ are Caputo's fractional derivatives, $f:[0 ; 1] \times \mathbb{R}^{3} \longrightarrow \mathbb{R}$ is a given continuous function, and

$$
\begin{aligned}
& \phi x(t)=\int_{0}^{t} \lambda(t, s) x(s) \mathrm{d} s, \\
& \psi x(t)=\int_{0}^{t} \delta(t, s) x(s) \mathrm{d} s
\end{aligned}
$$

where $\lambda, \delta:[0,1] \times[0,1] \longrightarrow[0,+\infty)$, with $\phi^{*}=\sup _{t \in[0,1]}$ $\left|\int_{0}^{t} \lambda(t, s) \mathrm{d} s\right|<\infty, \psi^{*}=\sup _{t \in[0,1]}\left|\int_{0}^{t} \delta(t, s) \mathrm{d} s\right|<\infty$.

Our motivation comes from the fact that not many papers have considered the existence and uniqueness results of nonlinear integro-differential equations with nonseparated boundary conditions. On top of that, we show the existence results under some weak conditions. The main results in this paper can be viewed as an extension of those provided in [22].

This paper is divided into five sections. In Section 2, we provide some notations and basic known results. In Section 3 , we study the new existence results to problem (1) under some weak conditions, and after that, we show the existence and uniqueness using Banach's contraction principle. In Section 4, we give two examples to illustrate the results. We end the paper with a conclusion.

\section{Preliminaries and Notations}

In this section, we state some notations, definitions, and lemmas which are used in this paper.

Definition 1 (see [5]). The fractional integral of order $\alpha>0$ with the lower limit zero for a function $f$ can be defined as

$$
I^{\alpha} f(t)=\frac{1}{\Gamma(\alpha)} \int_{0}^{t}(t-s)^{\alpha-1} f(s) \mathrm{d} s .
$$

Definition 2 (see [5]). The Caputo derivative of order $\alpha>0$ with the lower limit zero for a function $f$ can be defined as

$$
{ }^{c} D^{\alpha} f(t)=\frac{1}{\Gamma(n-\alpha)} \int_{0}^{t}(t-s)^{n-\alpha-1} f^{(n)}(s) \mathrm{d} s,
$$

where $n \in \mathbb{N}, 0 \leq n-1<\alpha<n, t>0$.

Theorem 1 (see [29]). Let $M$ be a bounded, closed, convex, and nonempty subset of a Banach space $X$. Let $A$ and $B$ be operators such that

(i) $A x+B y \in M$ whenever $x, y \in M$.

(ii) $A$ is compact and continuous.

(iii) $B$ is a contraction mapping.

Then, there exists $z \in M$ such that $z=A z+B z$.
A set of functions in $C[a, b]$ is relatively compact if and only it is uniformly bounded and equicontinuous on $[a, b]$.

Theorem 3 (see [30]). If a set is closed and relatively compact, then it is compact.

Lemma 1 (see [5]). Let $\alpha, \beta \geq 0$; then, the following relation holds:

$$
I^{\alpha} t^{\beta}=\frac{\Gamma(\beta+1)}{\Gamma(\alpha+\beta+1)} t^{\alpha+\beta} .
$$

Lemma 2 (see [5]). Let $n \in \mathbb{N}$ and $n-1<\alpha<n$. If $f$ is a continuous function, then we have

$$
I^{\alpha}\left({ }^{c} D^{\alpha} f(t)\right)=f(t)+a_{0}+a_{1} t+a_{2} t^{2}+\cdots+a_{n-1} t^{n-1} .
$$

Lemma 3. Let $y \in C([0,1], \mathbb{R})$. Then, a unique solution of the following boundary value problem:

$$
\left\{\begin{array}{l}
{ }^{c} D^{\alpha}\left({ }^{c} D^{\beta}\right) x(t)=y(t), \quad t \in[0,1] \\
x(0)=\lambda_{1} x(1) \\
x^{\prime}(0)=\lambda_{2} x^{\prime}(1) \\
{ }^{c} D^{\beta} x(0)=\lambda_{3}{ }^{c} D^{\beta} x(1) \\
{ }^{c} D^{\beta+1} x(0)=\lambda_{4}{ }^{c} D^{\beta+1} x(1)
\end{array}\right.
$$

is given by

$$
\begin{aligned}
x(t)= & \frac{1}{\Gamma(\alpha+\beta)} \int_{0}^{t}(t-s)^{\alpha+\beta-1} y(s) \mathrm{d} s+\frac{A_{1}(t)}{\Gamma(\alpha)} \\
& \cdot \int_{0}^{1}(1-s)^{\alpha-1} y(s) \mathrm{d} s \\
& +\frac{A_{2}(t)}{\Gamma(\alpha-1)} \int_{0}^{1}(1-s)^{\alpha-2} y(s) \mathrm{d} s+\frac{\lambda_{1}}{\left(1-\lambda_{1}\right) \Gamma(\alpha+\beta)} \\
& \cdot \int_{0}^{1}(1-s)^{\alpha+\beta-1} \times y(s) \mathrm{d} s \\
& +\frac{A_{3}(t)}{\Gamma(\alpha+\beta-1)} \int_{0}^{1}(1-s)^{\alpha+\beta-2} y(s) \mathrm{d} s
\end{aligned}
$$

where 


$$
\begin{aligned}
A_{1}(t)= & \frac{\lambda_{3}}{1-\lambda_{3}}\left(\frac{t^{\beta}}{\Gamma(\beta+1)}+\frac{\lambda_{1}}{\left(1-\lambda_{1}\right) \Gamma(\beta+1)}+\frac{\lambda_{2} \lambda_{1}}{\left(1-\lambda_{2}\right)\left(1-\lambda_{1}\right) \Gamma(\beta)}+\frac{t \lambda_{2}}{\left(1-\lambda_{2}\right) \Gamma(\beta)}\right) \\
A_{2}(t)= & \frac{\lambda_{4}}{1-\lambda_{4}}\left(\frac{t^{\beta} \lambda_{3}}{\Gamma(\beta+1)\left(1-\lambda_{3}\right)}+\frac{t^{\beta+1}}{\Gamma(\beta+2)}+\frac{\lambda_{2} \lambda_{1}}{\left(1-\lambda_{2}\right)\left(1-\lambda_{1}\right) \Gamma(\beta+1)}\right. \\
& +\frac{\lambda_{1}}{\left(1-\lambda_{1}\right) \Gamma(\beta+2)}+\frac{\lambda_{3} \lambda_{2} \lambda_{1}}{\left(1-\lambda_{3}\right)\left(1-\lambda_{2}\right)\left(1-\lambda_{1}\right) \Gamma(\beta)}+\frac{\lambda_{3} \lambda_{1}}{\left(1-\lambda_{3}\right)\left(1-\lambda_{1}\right) \Gamma(\beta+1)} \\
& \left.+\frac{\lambda_{3} \lambda_{2} t}{\left(1-\lambda_{3}\right)\left(1-\lambda_{2}\right) \Gamma(\beta)}+\frac{\lambda_{2}}{\left(1-\lambda_{2}\right) \Gamma(\beta+1)}\right) \\
A_{3}(t)= & \frac{\lambda_{2}}{1-\lambda_{2}}\left(t+\frac{\lambda_{1}}{1-\lambda_{1}}\right) .
\end{aligned}
$$

Proof. By Lemma 2, we have

$$
\begin{aligned}
{ }^{c} D^{\beta} x(t) & =I^{\alpha} y(t)+c_{0}+c_{1} t, \\
{ }^{c} D^{\beta+1} x(t) & =I^{\alpha-1} y(t)+c_{1}, \\
x(t) & =I^{\alpha+\beta} y(t)+I^{\beta} c_{0}+I^{\beta} c_{1} t+c_{2}+c_{3} t,
\end{aligned}
$$

where $c_{0}, c_{1}, c_{2}, c_{3} \in \mathbb{R}$. Using the condition ${ }^{c} D^{\beta+1} x(0)=\lambda_{4}{ }^{c} D^{\beta+1} x(1)$, we get

$$
c_{1}=\frac{\lambda_{4}}{\left(1-\lambda_{4}\right) \Gamma(\alpha-1)} \int_{0}^{1}(1-s)^{\alpha-2} y(s) \mathrm{d} s .
$$

By applying ${ }^{c} D^{\beta} x(0)=\lambda_{3}{ }^{c} D^{\beta} x(1)$, we have

$$
\begin{aligned}
c_{0}= & \frac{\lambda_{3}}{\left(1-\lambda_{3}\right) \Gamma(\alpha)} \int_{0}^{1}(1-s)^{\alpha-1} y(s) \mathrm{d} s \\
& +\frac{\lambda_{3} \lambda_{4}}{\left(1-\lambda_{4}\right)\left(1-\lambda_{3}\right) \Gamma(\alpha-1)}
\end{aligned}
$$

Now we use $x^{\prime}(0)=\lambda_{2} x^{\prime}(1)$ to get

$$
\begin{aligned}
c_{3}= & \frac{\lambda_{2}}{\left(1-\lambda_{2}\right) \Gamma(\alpha+\beta-1)} \int_{0}^{1}(1-s)^{\alpha+\beta-2} y(s) \mathrm{d} s \\
& +\frac{\lambda_{2} \lambda_{4}}{\left(1-\lambda_{4}\right)\left(1-\lambda_{2}\right) \Gamma(\beta+1) \Gamma(\alpha-1)} \int_{0}^{1}(1-s)^{\alpha-2} y(s) \mathrm{d} s \\
& +\frac{\lambda_{2} \lambda_{3} \lambda_{4}}{\left(1-\lambda_{4}\right)\left(1-\lambda_{3}\right)\left(1-\lambda_{2}\right) \Gamma(\beta) \Gamma(\alpha-1)} \int_{0}^{1}(1-s)^{\alpha-2} y(s) \mathrm{d} s \\
& +\frac{\lambda_{2} \lambda_{3}}{\left(1-\lambda_{3}\right)\left(1-\lambda_{2}\right) \Gamma(\alpha) \Gamma(\beta)} \int_{0}^{1}(1-s)^{\alpha-1} y(s) \mathrm{d} s .
\end{aligned}
$$


In view of $x(0)=\lambda_{1} x(1)$, we have

$$
\begin{aligned}
c_{2}= & \frac{\lambda_{1}}{\left(1-\lambda_{1}\right) \Gamma(\alpha+\beta)} \int_{0}^{1}(1-s)^{\alpha+\beta-1} y(s) \mathrm{d} s+\frac{\lambda_{1} \lambda_{2}}{\left(1-\lambda_{1}\right)\left(1-\lambda_{2}\right) \Gamma(\alpha+\beta-1)} \int_{0}^{1}(1-s)^{\alpha+\beta-2} y(s) \mathrm{d} s \\
& +\left(\frac{\lambda_{1} \lambda_{3}}{\left(1-\lambda_{1}\right)\left(1-\lambda_{3}\right) \Gamma(\beta+1)}+\frac{\lambda_{1} \lambda_{2} \lambda_{3}}{\left(1-\lambda_{1}\right)\left(1-\lambda_{2}\right)\left(1-\lambda_{3}\right) \Gamma(\beta)}\right) \\
& \times \frac{1}{\Gamma(\alpha)} \int_{0}^{1}(1-s)^{\alpha-1} y(s) \mathrm{d} s \\
& +\left(\frac{\lambda_{1} \lambda_{3} \lambda_{4}}{\left(1-\lambda_{1}\right)\left(1-\lambda_{3}\right)\left(1-\lambda_{4}\right) \Gamma(\beta+1)}+\frac{\lambda_{1} \lambda_{4}}{\left(1-\lambda_{1}\right)\left(1-\lambda_{4}\right) \Gamma(\beta+2)}\right. \\
& +\frac{\lambda_{1} \lambda_{2} \lambda_{4}}{\left(1-\lambda_{1}\right)\left(1-\lambda_{2}\right)\left(1-\lambda_{4}\right) \Gamma(\beta+1)} \\
& \left.+\frac{\lambda_{1} \lambda_{2} \lambda_{3} \lambda_{4}}{\left(1-\lambda_{1}\right)\left(1-\lambda_{2}\right)\left(1-\lambda_{3}\right)\left(1-\lambda_{4}\right) \Gamma(\beta)}\right) \frac{1}{\Gamma(\alpha-1)} \int_{0}^{1}(1-s)^{\alpha-2} y(s) \mathrm{d} s .
\end{aligned}
$$

Substituting the value of $c_{0}, c_{1}$, and $c_{2}$, we get the desired results. Directly computing, one can prove the converse of the lemma.

\section{Main Results}

Denote by $X$ the Banach space of all continuous functions from $[0,1] \longrightarrow \mathbb{R}$ endowed with the norms $\|x\|=\sup \{|x(t)|: t \in[0,1]\}$ and $\|y\|_{v}=\sup _{t \in[0,1]}\left(|y(t)| / e^{v t}\right)$, where $v>\left(1+\phi^{*}+\varphi^{*} / \Gamma(\alpha+\beta)\right)\|\sigma\|$, and $\sigma$ will be defined later.

By Lemma 3, we transform problem (1) into a fixed point problem as $x=P x$, where $P: X \longrightarrow X$ is given by

$$
\begin{aligned}
P x(t)= & \frac{1}{\Gamma(\alpha+\beta)} \int_{0}^{t}(t-s)^{\alpha+\beta-1} f(s, x(s), \phi x(s), \psi x(s)) \mathrm{d} s \\
& +\frac{A_{1}(t)}{\Gamma(\alpha)} \int_{0}^{1}(1-s)^{\alpha-1} f(s, x(s), \phi x(s), \psi x(s)) \mathrm{d} s \\
& +\frac{A_{2}(t)}{\Gamma(\alpha-1)} \int_{0}^{1}(1-s)^{\alpha-2} f(s, x(s), \phi x(s), \psi x(s)) \mathrm{d} s \\
& +\frac{\lambda_{1}}{\left(1-\lambda_{1}\right) \Gamma(\alpha+\beta)} \int_{0}^{1}(1-s)^{\alpha+\beta-1} f(s, x(s), \phi x(s), \psi x(s)) \mathrm{d} s \\
& +\frac{A_{3}(t)}{\Gamma(\alpha+\beta-1)} \int_{0}^{1}(1-s)^{\alpha+\beta-2} f(s, x(s), \phi x(s), \psi x(s)) \mathrm{d} s .
\end{aligned}
$$


For computational convenience, we set

$$
\begin{aligned}
A_{1}= & \frac{\left|\lambda_{3}\right|}{\left|1-\lambda_{3}\right|}\left(\frac{1}{\Gamma(\beta+1)}+\frac{\left|\lambda_{1}\right|}{\left|\left(1-\lambda_{1}\right)\right| \Gamma(\beta+1)}+\frac{\left|\lambda_{2} \lambda_{1}\right|}{\left|\left(1-\lambda_{2}\right)\left(1-\lambda_{1}\right)\right| \Gamma(\beta)}+\frac{\left|\lambda_{2}\right|}{\left|\left(1-\lambda_{2}\right)\right| \Gamma(\beta)}\right) \\
A_{2}= & \frac{\left|\lambda_{4}\right|}{\left|1-\lambda_{4}\right|}\left(\frac{\left|\lambda_{3}\right|}{\Gamma(\beta+1)\left|\left(1-\lambda_{3}\right)\right|}+\frac{1}{\Gamma(\beta+2)}+\frac{\left|\lambda_{2} \lambda_{1}\right|}{\left|\left(1-\lambda_{2}\right)\left(1-\lambda_{1}\right)\right| \Gamma(\beta+1)}\right. \\
& +\frac{\left|\lambda_{1}\right|}{\left|\left(1-\lambda_{1}\right)\right| \Gamma(\beta+2)}+\frac{\left|\lambda_{3} \lambda_{2} \lambda_{1}\right|}{\left|\left(1-\lambda_{3}\right)\left(1-\lambda_{2}\right)\left(1-\lambda_{1}\right)\right| \Gamma(\beta)} \\
& +\frac{\left|\lambda_{3} \lambda_{2}\right|}{\left|\left(1-\lambda_{3}\right)\left(1-\lambda_{1}\right)\right| \Gamma(\beta+1)}+\frac{\left|\left(1-\lambda_{3}\right)\left(1-\lambda_{2}\right)\right| \Gamma(\beta)}{\left|\lambda_{1}\right|} \\
& \left.+\frac{\left|\lambda_{2}\right|}{\left|\left(1-\lambda_{2}\right)\right| \Gamma(\beta+1)}\right) \\
A_{3}= & \frac{\left|\lambda_{2}\right|}{\left|1-\lambda_{2}\right|}\left(1+\frac{\left|\lambda_{1}\right|}{\left|1-\lambda_{1}\right|}\right) .
\end{aligned}
$$

Theorem 4. Suppose that

$\left(H_{1}\right)$ For all $t \in[0,1]$ and $x_{1}, x_{2}, x_{3}, y_{1}, y_{2}, y_{3} \in \mathbb{R}$, we have $\left|f\left(t, x_{1}, x_{2}, x_{3}\right)-f\left(t, y_{1}, y_{2}, y_{3}\right)\right| \leq \sigma(t)\left(\mid x_{1}-\right.$ $\left.y_{1}|+| x_{2}-y_{2}|+| x_{3}-y_{3} \mid\right)$ with $\sigma \in C([0,1] ;[t 0, \infty))$.
$\left(H_{2}\right) \quad|f(t, x, y, z)| \leq \theta(t), \forall(t, x, y, z) \in[0,1] \times \mathbb{R}^{3}$ with $\theta \in C\left([0,1] ; \mathbb{R}^{+}\right)$.

Then, problem (1) has at least one solution.

Proof. Define $B_{r^{\prime}}=\left\{x \in X:\|x\|_{v} \leq r^{\prime}\right\}$ with

$$
r^{\prime} \geq \frac{\|\theta\|_{v}\left(e^{v}-1\right)}{v}\left(\frac{1}{\Gamma(\alpha+\beta)}+\frac{A_{1}}{\Gamma(\alpha)}+\frac{A_{2}}{\Gamma(\alpha-1)}+\frac{\left|\lambda_{1}\right|}{\left|1-\lambda_{1}\right| \Gamma(\alpha+\beta)}+\frac{A_{3}}{\Gamma(\alpha+\beta-1)}\right) .
$$

We introduce the decomposition $P=P_{1}+P_{2}$, where

$$
\begin{aligned}
P_{1} x(t)= & \frac{1}{\Gamma(\alpha+\beta)} \int_{0}^{t}(t-s)^{\alpha+\beta-1} f(s, x(s), \phi x(s), \psi x(s)) \mathrm{d} s \\
P_{2} x(t)= & \frac{A_{1}(t)}{\Gamma(\alpha)} \int_{0}^{1}(1-s)^{\alpha-1} f(s, x(s), \phi x(s), \psi x(s)) \mathrm{d} s \\
& +\frac{A_{2}(t)}{\Gamma(\alpha-1)} \int_{0}^{1}(1-s)^{\alpha-2} f(s, x(s), \phi x(s), \psi x(s)) \mathrm{d} s \\
& +\frac{\lambda_{1}}{\left(1-\lambda_{1}\right) \Gamma(\alpha+\beta)} \int_{0}^{1}(1-s)^{\alpha+\beta-1} f(s, x(s), \phi x(s), \psi x(s)) \mathrm{d} s \\
& +\frac{A_{3}(t)}{\Gamma(\alpha+\beta-1)} \int_{0}^{1}(1-s)^{\alpha+\beta-2} f(s, x(s), \phi x(s), \psi x(s)) \mathrm{d} s .
\end{aligned}
$$


6

Mathematical Problems in Engineering

For $x, y \in B_{r^{\prime}}$, we have

$$
\begin{aligned}
& \left\|P_{1} x(t)\right\|_{v} \leq \sup _{t \in[0,1]} \frac{1}{e^{v t}}\left|\frac{1}{\Gamma(\alpha+\beta)} \int_{0}^{t}(t-s)^{\alpha+\beta-1} f(s, x(s), \phi x(s), \psi x(s)) \mathrm{d} s\right| \\
& \leq \sup _{t \in[0,1]} \frac{1}{e^{v t}} \frac{1}{\Gamma(\alpha+\beta)} \int_{0}^{t}(t-s)^{\alpha+\beta-1}|\theta(s)| \mathrm{d} s \\
& \leq \sup _{t \in[0,1]} \frac{1}{e^{v t}} \frac{1}{\Gamma(\alpha+\beta)} \int_{0}^{t}(t-s)^{\alpha+\beta-1} \frac{|\theta(s)| e^{v s}}{e^{v s}} \mathrm{~d} s \\
& \leq \sup _{t \in[0,1]} \frac{\|\theta\|_{v}}{e^{v t} \Gamma(\alpha+\beta)} \int_{0}^{t}(t-s)^{\alpha+\beta-1} e^{v s} \mathrm{~d} s \\
& \leq \sup _{t \in[0,1]} \frac{\|\theta\|_{v}}{e^{v t} \Gamma(\alpha+\beta)} \int_{0}^{t} e^{v s} \mathrm{~d} s \\
& \leq \sup _{t \in[0,1]} \frac{\|\theta\|_{v}}{v \Gamma(\alpha+\beta)} \frac{e^{v t}-1}{e^{v t}} \\
& \leq \frac{\|\theta\|_{v}\left(e^{v}-1\right)}{v \Gamma(\alpha+\beta)} \\
& \left\|P_{2} y(t)\right\|_{v} \leq \sup _{t \in[0,1]} \frac{1}{e^{v t}} \mid \frac{A_{1}(t)}{\Gamma(\alpha)} \int_{0}^{1}(1-s)^{\alpha-1} f(s, y(s), \phi y(s), \psi y(s)) \mathrm{d} s \\
& +\frac{A_{2}(t)}{\Gamma(\alpha-1)} \int_{0}^{1}(1-s)^{\alpha-2} f(s, y(s), \phi y(s), \psi y(s)) \mathrm{d} s \\
& +\frac{\lambda_{1}}{\left(1-\lambda_{1}\right) \Gamma(\alpha+\beta)} \int_{0}^{1}(1-s)^{\alpha+\beta-1} f(s, y(s), \phi y(s), \psi y(s)) \mathrm{d} s \\
& +\frac{A_{3}(t)}{\Gamma(\alpha+\beta-1)} \int_{0}^{1}(1-s)^{\alpha+\beta-2} f(s, y(s), \phi y(s), \psi y(s)) \mathrm{d} s \mid \\
& \leq \sup _{t \in[0,1]} \frac{1}{e^{v t}}\left[\frac{A_{1}}{\Gamma(\alpha)} \int_{0}^{1}(1-s)^{\alpha-1} \frac{|\theta(s)| e^{v s}}{e^{v s}} \mathrm{~d} s+\frac{A_{2}}{\Gamma(\alpha-1)} \int_{0}^{1}(1-s)^{\alpha-2} \times \frac{|\theta(s)| e^{v s}}{e^{v s}} \mathrm{~d} s\right. \\
& \left.+\frac{\left|\lambda_{1}\right|}{\left|1-\lambda_{1}\right| \Gamma(\alpha+\beta)} \int_{0}^{1}(1-s)^{\alpha+\beta-1} \frac{|\theta(s)| e^{v s}}{e^{v s}} \mathrm{~d} s+\frac{A_{3}}{\Gamma(\alpha+\beta-1)} \int_{0}^{1}(1-s)^{\alpha+\beta-2} \frac{|\theta(s)| e^{v s}}{e^{v s}} \mathrm{~d} s\right] \\
& \leq \sup _{t \in[0,1]} \frac{\|\theta\|_{v}}{e^{v t}}\left[\frac{A_{1}}{\Gamma(\alpha)} \int_{0}^{1}(1-s)^{\alpha-1} e^{v s} \mathrm{~d} s+\frac{A_{2}}{\Gamma(\alpha-1)} \int_{0}^{1}(1-s)^{\alpha-2} e^{v s} \mathrm{~d} s\right. \\
& \left.+\frac{\left|\lambda_{1}\right|}{\left|1-\lambda_{1}\right| \Gamma(\alpha+\beta)} \int_{0}^{1}(1-s)^{\alpha+\beta-1} e^{v s} \mathrm{~d} s+\frac{A_{3}}{\Gamma(\alpha+\beta-1)} \int_{0}^{1}(1-s)^{\alpha+\beta-2} e^{v s} \mathrm{~d} s\right] \\
& \leq \sup _{t \in[0,1]} \frac{\|\theta\|_{v}}{e^{v t}}\left[\frac{A_{1}}{\Gamma(\alpha)} \int_{0}^{1} e^{v s} \mathrm{~d} s+\frac{A_{2}}{\Gamma(\alpha-1)} \int_{0}^{1} e^{v s} \mathrm{~d} s+\frac{\left|\lambda_{1}\right|}{\left|1-\lambda_{1}\right| \Gamma(\alpha+\beta)} \times \int_{0}^{1} e^{v s} \mathrm{~d} s+\frac{A_{3}}{\Gamma(\alpha+\beta-1)} \int_{0}^{1} e^{v s} \mathrm{~d} s\right]
\end{aligned}
$$

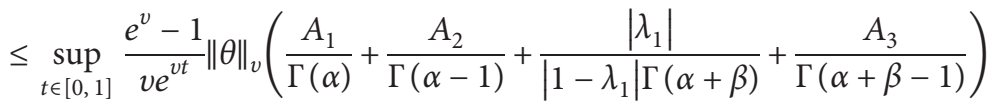

$$
\begin{aligned}
& \leq \frac{e^{v}-1}{v}\|\theta\|_{v}\left(\frac{A_{1}}{\Gamma(\alpha)}+\frac{A_{2}}{\Gamma(\alpha-1)}+\frac{\left|\lambda_{1}\right|}{\left|1-\lambda_{1}\right| \Gamma(\alpha+\beta)}+\frac{A_{3}}{\Gamma(\alpha+\beta-1)}\right) .
\end{aligned}
$$


Thus,

$$
\left\|P_{1} x+P_{2} y\right\|_{v} \leq \frac{\|\theta\|_{v}\left(e^{v}-1\right)}{v}\left(\frac{1}{\Gamma(\alpha+\beta)}+\frac{A_{1}}{\Gamma(\alpha)}+\frac{A_{2}}{\Gamma(\alpha-1)}+\frac{\left|\lambda_{1}\right|}{\left|1-\lambda_{1}\right| \Gamma(\alpha+\beta)}+\frac{A_{3}}{\Gamma(\alpha+\beta-1)}\right) .
$$

Hence, $P_{1} x+P_{2} y \in B_{r^{\prime}}$.

For $x, y \in B_{r^{\prime}}$, consider

$$
\begin{aligned}
\left\|P_{1} y(t)-P_{1} x(t)\right\|_{v} \leq & \sup _{t \in[0,1]} \frac{1}{\Gamma(\alpha+\beta) e^{v t}} \int_{0}^{t}(t-s)^{\alpha+\beta-1} \\
& \times|f(s, y(s), \phi y(s), \psi y(s))-f(s, x(s), \phi x(s), \psi x(s))| \mathrm{d} s \\
\leq & \sup _{t \in[0,1]} \frac{1}{\Gamma(\alpha+\beta) e^{v t}} \int_{0}^{t}(t-s)^{\alpha+\beta-1} \sigma(s)(|y(s)-x(s)|+|\phi y(s)-\phi x(s)|+|\psi y(s)-\psi x(s)|) \mathrm{d} s \\
\leq & \sup _{t \in[0,1]} \frac{\|\sigma\|}{\Gamma(\alpha+\beta) e^{v t}} \int_{0}^{t} e^{v s}\left(\|y-x\|_{v}+\phi^{*}\|y-x\|_{v}+\psi^{*}\|y-x\|_{v}\right) \mathrm{d} s \\
\leq & \sup _{t \in[0,1]} \frac{\left(1+\phi^{*}+\psi^{*}\right)\|\sigma\| e^{v t}-1}{v \Gamma(\alpha+\beta)}\|y-x\|_{v} \\
\leq & \frac{\left(1+\phi^{v t} \| \psi^{*}\right)\|\sigma\|\|y-x\|_{v}}{\Gamma(\alpha+\beta) v} .
\end{aligned}
$$

By using the condition of the new norm, we have that $P_{1}$ is a contraction.

Next, we will show that $P_{2}$ is compact and continuous.

$$
\left\|P_{2} y\right\|_{v} \leq \frac{e^{v}-1}{v}\|\theta\|_{v}\left(\frac{A_{1}}{\Gamma(\alpha)}+\frac{A_{2}}{\Gamma(\alpha-1)}+\frac{\left|\lambda_{1}\right|}{\left|1-\lambda_{1}\right| \Gamma(\alpha+\beta)}+\frac{A_{3}}{\Gamma(\alpha+\beta-1)}\right) .
$$

Suppose that $0 \leq t_{1}<t_{2} \leq 1$. We have

$$
\begin{aligned}
\left|P_{2} y\left(t_{2}\right)-P_{2} y\left(t_{1}\right)\right| \leq & \frac{\left|A_{1}\left(t_{2}\right)-A_{1}\left(t_{1}\right)\right|}{\Gamma(\alpha)} \int_{0}^{1}(1-s)^{\alpha-1}|f(s, y(s), \phi y(s), \psi y(s))| \\
& \times \mathrm{d} s+\frac{\left|A_{1}\left(t_{2}\right)-A_{1}\left(t_{1}\right)\right|}{\Gamma(\alpha-1)} \int_{0}^{1}(1-s)^{\alpha-2} \\
& \times|f(s, y(s), \phi y(s), \psi y(s))| \mathrm{d} s+\frac{\left|A_{3}\left(t_{2}\right)-A_{3}\left(t_{1}\right)\right|}{\Gamma(\alpha+\beta-1)} \\
& \times \int_{0}^{1}(1-s)^{\alpha+\beta-2}|f(s, y(s), \phi y(s), \psi y(s))| \mathrm{d} s .
\end{aligned}
$$


Thus,

$\left|P_{2} y\left(t_{2}\right)-P_{2} y\left(t_{1}\right)\right| \longrightarrow 0, \quad$ as $t_{1} \longrightarrow t_{2}$ independently from $y \in B_{r^{\prime}}$.

This proves that the operator $P_{2}$ is relatively compact on $B_{r^{\prime}}$. Then, by the Arzelá-Ascoli theorem, we have that $P_{2}$ is compact on $B_{r^{\prime}}$. $B_{r^{\prime}}$

Therefore, problem (1) has at least one solution on

$$
r_{1}=\left(1+\phi^{*}+\psi^{*}\right) \Sigma^{*}\left(\frac{1}{\Gamma(\alpha+\beta)}+\frac{A_{1}}{\Gamma(\alpha)}+\frac{A_{2}}{\Gamma(\alpha-1)}+\frac{\left|\lambda_{1}\right|}{\left|\left(1-\lambda_{1}\right)\right| \Gamma(\alpha+\beta)}+\frac{A_{3}}{\Gamma(\alpha+\beta-1)}\right)<1 .
$$

Proof. Set $\sup _{0 \leq t \leq 1}|f(t, 0,0,0)|=M$.

Let $B_{r}=\{x \in X:\|x\| \leq r\}$ be the ball with radius $r$, where $r \geq\left(r_{2} / 1-r_{1}\right)$, with

$$
\begin{aligned}
r_{2}= & \frac{M}{\Gamma(\alpha+\beta)}+\frac{A_{1} M}{\Gamma(\alpha)}+\frac{A_{2} M}{\Gamma(\alpha-1)} \\
& +\frac{\left|\lambda_{1}\right| M}{\left|\left(1-\lambda_{1}\right)\right| \Gamma(\alpha+\beta)}+\frac{A_{3} M}{\Gamma(\alpha+\beta-1)} .
\end{aligned}
$$

Theorem 5. Assume that $f:[0,1] \times \mathbb{R}^{3} \longrightarrow \mathbb{R}$ is continuous function satisfying

$\left(H_{1}\right)$ For all $t \in[0,1]$ and $x_{1}, x_{2}, x_{3}, y_{1}, y_{2}, y_{3} \in \mathbb{R}$, we have $\left|f\left(t, x_{1}, x_{2}, x_{3}\right)-f\left(t, y_{1}, y_{2}, y_{3}\right)\right| \leq \Sigma(t) \quad\left(\mid x_{1}-\right.$ $\left.y_{1}|+| x_{2}-y_{2}|+| x_{3}-y_{3} \mid\right)$ with $\Sigma \in L^{1}([0,1] ;[t 0, \infty))$ and $\Sigma^{*}=\int_{0}^{1} \Sigma(s) d s$.

Then, there exists a unique solution for the boundary value problem (1) provided that

Then, $B_{r}$ is a closed, convex, and nonempty subset of the Banach space $X$.

Our objective is to show that the operator $P$ has a unique fixed point on $B_{r}$.

We prove that $P B_{r} \subseteq B_{r}$.

For $x \in B_{r}, t \in[0,1]$, we have

$$
\begin{aligned}
& |P x(t)| \leq \frac{1}{\Gamma(\alpha+\beta)} \int_{0}^{t}(t-s)^{\alpha+\beta-1}|f(s, x(s), \phi x(s), \psi x(s))| \mathrm{d} s \\
& +\frac{\left|A_{1}(t)\right|}{\Gamma(\alpha)} \int_{0}^{1}(1-s)^{\alpha-1}|f(s, x(s), \phi x(s), \psi x(s))| \mathrm{d} s \\
& +\frac{\left|A_{2}(t)\right|}{\Gamma(\alpha-1)} \int_{0}^{1}(1-s)^{\alpha-2}|f(s, x(s), \phi x(s), \psi x(s))| \mathrm{d} s \\
& +\frac{\left|\lambda_{1}\right|}{\left|\left(1-\lambda_{1}\right)\right| \Gamma(\alpha+\beta)} \int_{0}^{1}(1-s)^{\alpha+\beta-1}|f(s, x(s), \phi x(s), \psi x(s))| \mathrm{d} s \\
& +\frac{\left|A_{3}(t)\right|}{\Gamma(\alpha+\beta-1)} \int_{0}^{1}(1-s)^{\alpha+\beta-2}|f(s, x(s), \phi x(s), \psi x(s))| \mathrm{d} s \\
& \leq \frac{1}{\Gamma(\alpha+\beta)} \int_{0}^{t}(t-s)^{\alpha+\beta-1}(|f(s, x(s), \phi x(s), \psi x(s))-f(s, 0,0,0)|+|f(s, 0,0,0)|) \mathrm{d} s \\
& +\frac{\left|A_{1}(t)\right|}{\Gamma(\alpha)} \int_{0}^{1}(1-s)^{\alpha-1}(|f(s, x(s), \phi x(s), \psi x(s))-f(s, 0,0,0)|+|f(s, 0,0,0)|) \mathrm{d} s \\
& +\frac{\left|A_{2}(t)\right|}{\Gamma(\alpha-1)} \int_{0}^{1}(1-s)^{\alpha-2} \times(|f(s, x(s), \phi x(s), \psi x(s))-f(s, 0,0,0)|+|f(s, 0,0,0)|) \mathrm{d} s \\
& +\frac{\left|\lambda_{1}\right|}{\left|\left(1-\lambda_{1}\right)\right| \Gamma(\alpha+\beta)} \int_{0}^{1}(1-s)^{\alpha+\beta-1}(|f(s, x(s), \phi x(s), \psi x(s))-f(s, 0,0,0)|+|f(s, 0,0,0)|) \mathrm{d} s \\
& +\frac{\left|A_{3}(t)\right|}{\Gamma(\alpha+\beta-1)} \\
& \left.\times \int_{0}^{1}(1-s)^{\alpha+\beta-2}|f(s, x(s), \phi x(s), \psi x(s))-f(s, 0,0,0)|+|f(s, 0,0,0)|\right) \mathrm{d} s
\end{aligned}
$$


Mathematical Problems in Engineering

9

$$
\begin{aligned}
& \leq \frac{1}{\Gamma(\alpha+\beta)} \int_{0}^{t}(t-s)^{\alpha+\beta-1}(\Sigma(s)(|x(s)|+|\phi x(s)|+|\psi x(s)|)+M) \mathrm{d} s \\
& +\frac{A_{1}}{\Gamma(\alpha)} \int_{0}^{1}(1-s)^{\alpha-1}(\Sigma(s)(|x(s)|+|\phi x(s)|+|\psi x(s)|)+M) \mathrm{d} s \\
& +\frac{A_{2}}{\Gamma(\alpha-1)} \int_{0}^{1}(1-s)^{\alpha-2}(\Sigma(s)(|x(s)|+|\phi x(s)|+|\psi x(s)|)+M) \mathrm{d} s \\
& +\frac{\left|\lambda_{1}\right|}{\left|\left(1-\lambda_{1}\right)\right| \Gamma(\alpha+\beta)} \int_{0}^{1}(1-s)^{\alpha+\beta-1}(\Sigma(s)(|x(s)|+|\phi x(s)|+|\psi x(s)|)+M) \mathrm{d} s \\
& +\frac{A_{3}}{\Gamma(\alpha+\beta-1)} \int_{0}^{1}(1-s)^{\alpha+\beta-2}(\Sigma(s) \times(|x(s)|+|\phi x(s)|+|\psi x(s)|)+M) \mathrm{d} s \\
& \leq \frac{\left(1+\phi^{*}+\psi^{*}\right)\|x\|}{\Gamma(\alpha+\beta)} \int_{0}^{1} \Sigma(s) \mathrm{d} s+\frac{M}{\Gamma(\alpha+\beta)} \int_{0}^{t}(t-s)^{\alpha+\beta-1} \mathrm{~d} s+\frac{\left(1+\phi^{*}+\psi^{*}\right) A_{1}\|x\|}{\Gamma(\alpha)} \int_{0}^{1} \Sigma(s) \mathrm{d} s \\
& +\frac{A_{1} M}{\Gamma(\alpha)} \int_{0}^{t}(t-s)^{\alpha-1} \mathrm{~d} s+\frac{\left(1+\phi^{*}+\psi^{*}\right) A_{2}\|x\|}{\Gamma(\alpha-1)} \int_{0}^{1} \Sigma(s) \mathrm{d} s+\frac{A_{2} M}{\Gamma(\alpha-1)} \int_{0}^{t}(t-s)^{\alpha-2} \mathrm{~d} s \\
& +\frac{\left|\lambda_{1}\right|\left(1+\phi^{*}+\psi^{*}\right)\|x\|}{\left|\left(1-\lambda_{1}\right)\right| \Gamma(\alpha+\beta)} \int_{0}^{1} \Sigma(s) \mathrm{d} s+\frac{\left|\lambda_{1}\right| M}{\left|\left(1-\lambda_{1}\right)\right| \Gamma(\alpha+\beta)} \int_{0}^{1}(1-s)^{\alpha+\beta-1} \mathrm{~d} s \\
& +\frac{A_{3}\left(1+\phi^{*}+\psi^{*}\right)\|x\|}{\Gamma(\alpha+\beta-1)} \int_{0}^{1} \Sigma(s) \mathrm{d} s+\frac{A_{3} M}{\Gamma(\alpha+\beta-1)} \int_{0}^{1}(1-s)^{\alpha+\beta-2} \mathrm{~d} s,
\end{aligned}
$$

which implies that

$$
\begin{aligned}
\|P x\| \leq & \frac{\left(1+\phi^{*}+\psi^{*}\right) \Sigma^{*}\|x\|}{\Gamma(\alpha+\beta)}+\frac{M}{\Gamma(\alpha+\beta)} \\
& +\frac{\left(1+\phi^{*}+\psi^{*}\right) \Sigma^{*} A_{1}\|x\|}{\Gamma(\alpha)}+\frac{A_{1} M}{\Gamma(\alpha)} \\
& +\frac{\left(1+\phi^{*}+\psi^{*}\right) \Sigma^{*} A_{2}\|x\|}{\Gamma(\alpha-1)}+\frac{A_{2} M}{\Gamma(\alpha-1)} \\
& +\frac{\left|\lambda_{1}\right|\left(1+\phi^{*}+\psi^{*}\right) \Sigma^{*}\|x\|}{\left|\left(1-\lambda_{1}\right)\right| \Gamma(\alpha+\beta)}+\frac{\left|\lambda_{1}\right| M}{\left|\left(1-\lambda_{1}\right)\right| \Gamma(\alpha+\beta)} \\
& +\frac{A_{3}\left(1+\phi^{*}+\psi^{*}\right) \Sigma^{*}\|x\|}{\Gamma(\alpha+\beta-1)}+\frac{A_{3} M}{\Gamma(\alpha+\beta-1)} \\
\leq & {\left[\frac{\left(1+\phi^{*}+\psi^{*}\right) \Sigma^{*}}{\Gamma(\alpha+\beta)}+\frac{\left(1+\phi^{*}+\psi^{*}\right) \Sigma^{*} A_{1}}{\Gamma(\alpha)}+\frac{\left(1+\phi^{*}+\psi^{*}\right) \Sigma^{*} A_{2}}{\Gamma(\alpha-1)}+\frac{\left|\lambda_{1}\right|\left(1+\phi^{*}+\psi^{*}\right) \Sigma^{*}}{\left|\left(1-\lambda_{1}\right)\right| \Gamma(\alpha+\beta)}+\frac{A_{3}\left(1+\phi^{*}+\psi^{*}\right) \Sigma^{*}}{\Gamma(\alpha+\beta-1)}\right] } \\
\leq & \|x\| \\
\leq & r_{1} r+r_{2} \\
\Gamma(\alpha+\beta) & \frac{A_{1} M}{\Gamma(\alpha)}+\frac{A_{2} M}{\Gamma(\alpha-1)}+\frac{A_{3} M}{\left|\left(1-\lambda_{1}\right)\right| \Gamma(\alpha+\beta)}+\frac{\left.\lambda_{1} \mid M+1\right)}{\Gamma(\alpha+\beta-1)}
\end{aligned}
$$


Now, for $x, y \in B_{r}$ and for $t \in[0,1]$,

$$
\begin{aligned}
|P x(t)-P y(t)| \leq & \frac{1}{\Gamma(\alpha+\beta)} \int_{0}^{t}(t-s)^{\alpha+\beta-1}|f(s, x(s), \phi x(s), \psi x(s))-f(s, y(s), \phi y(s), \psi y(s))| \mathrm{d} s \\
& +\frac{\left|A_{1}(t)\right|}{\Gamma(\alpha)} \int_{0}^{1}(1-s)^{\alpha-1} \times|f(s, x(s), \phi x(s), \psi x(s))-f(s, y(s), \phi y(s), \psi y(s))| \mathrm{d} s \\
& +\frac{\left|A_{2}(t)\right|}{\Gamma(\alpha-1)} \int_{0}^{1}(1-s)^{\alpha-2}|f(s, x(s), \phi x(s), \psi x(s))-f(s, y(s), \phi y(s), \psi y(s))| \mathrm{d} s \\
& +\frac{\left|\left(1-\lambda_{1}\right)\right| \Gamma(\alpha+\beta)}{\left|\lambda_{1}\right|} \\
& \times \int_{0}^{1}(1-s)^{\alpha+\beta-1}|f(s, x(s), \phi x(s), \psi x(s))-f(s, y(s), \phi y(s), \psi y(s))| \mathrm{d} s \\
& +\frac{\left|A_{3}(t)\right|}{\Gamma(\alpha+\beta-1)} \\
& \times \int_{0}^{1}(1-s)^{\alpha+\beta-2}|f(s, x(s), \phi x(s), \psi x(s))-f(s, y(s), \phi y(s), \psi y(s))| \mathrm{d} s,
\end{aligned}
$$

and then

$$
\|P x-P y\| \leq r_{1}\|x-y\| .
$$

Since $r_{1}<1$, then the operator $P$ is a contraction mapping. Thus, problem (1) has a unique solution.

\section{Examples}

Example 1. Consider the following fractional problem:

$$
\left\{\begin{array}{l}
{ }^{c} D^{(17 / 11)}\left({ }^{c} D^{(15 / 11)}\right) x(t)=\frac{t^{4}}{800}\left(\frac{|x(t)| e^{-t}}{1+|x(t)|}+\int_{0}^{t} \frac{(t+s)^{4}|x(s)|\left(e^{-2 s}+e^{-3 s}\right)}{800(1+|x(s)|)} \mathrm{d} s\right), \quad t \in[0,1], \\
x(0)=\frac{1}{200} x(1), \\
x^{\prime}(0)=\frac{1}{200} x^{\prime}(1), \\
{ }^{c} D^{(15 / 11)} x(0)=\frac{1}{200}{ }^{c} D^{(15 / 11)} x(1), \\
{ }^{c} D^{(26 / 11)} x(0)=\frac{1}{200}{ }^{c} D^{(26 / 11)} x(1) .
\end{array}\right.
$$


We choose

$$
\begin{aligned}
\beta & =\frac{15}{11}, \\
\alpha & =\frac{17}{11}, \\
f(t, x, y, z) & =\frac{t^{4}}{800}\left(\frac{|x(t)| e^{-t}}{1+|x(t)|}+\frac{|y(t)| e^{-2 t}}{1+|y(t)|}+\frac{|z(t)| e^{-3 t}}{1+|z(t)|}\right), \\
\lambda(t, s) & =\delta(t, s)=\frac{(t+s)^{4}}{800} .
\end{aligned}
$$

$\left|f\left(t, x_{1}, x_{2}, x_{3}\right)-f\left(t, y_{1}, y_{2}, y_{3}\right)\right| \leq \frac{t^{4}}{800}\left(\left|x_{1}-y_{1}\right|+\left|x_{2}-y_{2}\right|+\left|x_{3}-y_{3}\right|\right), \quad$ for all $t \in[0,1]$ and $x_{1}, x_{2}, x_{3}, y_{1}, y_{2}, y_{3} \in \mathbb{R}$

$$
\left|f\left(t, x_{1}, x_{2}, x_{3}\right)\right| \leq \frac{3 t^{4}}{800}
$$

Clearly, $\phi^{*}=\psi^{*}=(31 / 4000),\|\sigma\|=(1 / 800)$.

Hence, by Theorem 4, problem (31) has a least one solution.$$
\left|f\left(t, x_{1}, x_{2}, x_{3}\right)\right| \leq \frac{3 t}{800}
$$

Example 2. Consider the following boundary value problem:
We have

$$
\left\{\begin{array}{l}
{ }^{c} D^{(10 / 6)}\left({ }^{c} D^{(8 / 6)}\right) x(t)=\frac{t^{3}}{3000}\left(\frac{1}{1+|x(t)|}+\frac{1}{1000} \int_{0}^{t} t^{5} s^{4} x(s) \mathrm{d} s\right), \quad t \in[0,1] \\
x(0)=\frac{1}{201} x(1), \\
x^{\prime}(0)=\frac{1}{201} x^{\prime}(1), \\
{ }^{c} D^{(8 / 6)} x(0)=\frac{1}{201}{ }^{c} D^{(8 / 6)} x(1), \\
{ }^{c} D^{(14 / 6)} x(0)=\frac{1}{201}{ }^{c} D^{(14 / 6)} x(1) .
\end{array}\right.
$$

Here $\beta=(8 / 6), \quad \alpha=(10 / 6), \quad f(t, x, y, z)=\left(t^{3} / 3000\right)$ $((1 / 1+|x(t)|)+y(t)+z(t)), \lambda(t, s)=\delta(t, s)=\left(t^{5} s^{4} / 2000\right)$.

We get

$$
\left|f\left(t, x_{1}, x_{2}, x_{3}\right)-f\left(t, y_{1}, y_{2}, y_{3}\right)\right| \leq \frac{t^{3}}{3000}\left(\left|x_{1}-y_{1}\right|+\left|x_{2}-y_{2}\right|+\left|x_{3}-y_{3}\right|\right), \quad \text { for all } t \in[0,1] \text { and } x_{1}, x_{2}, x_{3}, y_{1}, y_{2}, y_{3}, \in \mathbb{R} \text {. }
$$


Clearly, $\phi^{*}=\psi^{*}=(1 / 10000), \Sigma^{*}=(1 / 12000)$.

Hence, we have $r_{1} \approx 0.000043<1$.

Then, problem (34) has a unique solution.

\section{Conclusion}

In this paper, new existence and uniqueness results have been studied for nonlinear fractional integro-differential equations equipped with nonseparated conditions. By using a new norm, we have established the new existence of solution for (1) under some weak conditions. The uniqueness of solutions has been given by using the sup norm and applying the Banach fixed point theorem. We have ended the article with some examples to illustrate the results.

\section{Data Availability}

No data were used to support this study.

\section{Conflicts of Interest}

The authors declare that there are no conflicts of interest regarding the publication of this paper.

\section{References}

[1] V. Lakshmikantham, "Theory of fractional functional differential equations," Nonlinear Analysis, vol. 69, no. 10, pp. 3337-3343, 2007.

[2] V. Lakshmikantham and A. S. Vatsala, "Basic theory of fractional differential equations," Nonlinear Analysis, vol. 69, no. 8, pp. 2677-2682, 2007.

[3] K. S. Miller and B. Ross, An Introduction to the Fractional Calculus and Fractional Differential Equations, Wiley, New York, NY, USA, 1993.

[4] R. Hilfer, Applications of Fractional Calculs in Physics, World Scientific, Singapore, 2000.

[5] I. Podlubny, Fractional Differential Equations, Academic Press, New York, NY, USA, 1993.

[6] Y. Zhou, Basic Theory of Fractional Differential Equations, Xiangtan University, Xiangtan, China, 2014.

[7] A. A. Kilbas, H. M. Srivastava, and J. J. Trujillo, Theory and Applications of Fractional Differential Equations, NorthHolland Mathematics Studies, Vol. 204, Elsevier, Amsterdam, Netherlands, 2006.

[8] K. Hilal, L. Ibnelazyz, K. Guida, and S. Melliani, Existence of Mild Solutions for an Impulsive Fractional Integro-differential Equations with Non-local Condition, Springer Nature Switzerland AG, New York, NY, USA, 2019.

[9] K. Hilal, K. Guida, L. Ibnelazyz, and M. Oukessou, Existence Results for an Impulsive Fractional Integro-differential Equations with Non-compact Semigroup, Springer Nature Switzerland AG, New York, NY, USA, 2019.

[10] K. Guida, K. Hilal, and L. Ibnelazyz, "Existence of mild solutions for a class of impulsive hilfer fractional coupled systems," Advances in Mathematical Physics, vol. 2020, Article ID 8406509, 2020.

[11] L. Ibnelazyz, K. Guida, K. Hilal, and S. Melliani, "Existence of solution for a fractional Langevin system with nonseparated integral boundary conditions," Journal of Mathematics, vol. 2021, Article ID 3482153, 2021.
[12] L. Ibnelazyz, K. Guida, K. Hilal, and S. Melliani, "New existence results for nonlinear fractional integrodifferential equations," Advances in Mathematical Physics, vol. 2021, Article ID 5525591, 2021.

[13] L. Ibnelazyz, K. Guida, K. Hilal, and S. Melliani, "Existence results for nonlinear fractional integro-differential equations with integral and antiperiodic boundary conditions," Computational and Applied Mathematics, vol. 40, p. 33, 2021.

[14] D. Baleanu, K. Ghafarnezhad, and S. Rezapour, "On a three step crisis integro-differential equation," Advances in Difference Equations, vol. 1, p. 153, 2019.

[15] D. Baleanu, G. Khadijeh, R. Shahram, and S. Mehdi, "On the existence of solutions of a three steps crisis integro-differential, equation," Advances in Difference Equations, vol. 1, p. 135, 2018.

[16] B. Ahmad, S. K. Ntouyas, R. Agarwal, and A. Alsaedi, "Existence results for sequential fractional integro-differential equations with nonlocal multi-point and strip conditions," Boundary Value Problems, vol. 2016, p. 205, 2016.

[17] L. Ibnelazyz, K. Guida, S. Melliani, and K. Hilal, "On a nonlocal multipoint and integral boundary value problem of nonlinear fractional integrodifferential equations," Journal of Function Spaces, vol. 2020, Article ID 8891736, 2020.

[18] B. Ahmed and S. Sivasundaram, "On a four-point nonlocal boundary value problem of nonlinear integro-differential equations of fractional order," Applied Mathematics and Computation, vol. 217, pp. 480-487, 2010.

[19] D. Baleanu, S. Z. Nazeni, and S. Rezapour, "Existence and uniqueness of solutions for multi-term nonlinear fractional integro-differential equations," Advances in Difference Equations, vol. 2013, p. 368, 2013.

[20] Y. Wang and L. Liu, "Uniqueness and existence of positive solutions for the fractional integro-differential equation," Boundary Value Problems, vol. 12, 2017.

[21] A. Ahmed and B. Ahmad, "Existence of solutions for nonlinear fractional integro-differential equations with threepoint nonlocal fractional boundary conditions," Advances in Difference Equations, vol. 2010, Article ID 691721, 2020.

[22] A. Bragdi, A. Frioui, and A. G. Lakoud, "Existence of solutions for nonlinear fractional integro-differential equations," $A d$ vances in Difference Equations, vol. 2020, p. 418, 2020.

[23] K. Hilal, L. Ibnelazyz, K. Guida, and S. Melliani, "Fractional Langevin equations with nonseparated integral boundary conditions," Advances in Mathematical Physics, vol. 2020, Article ID 3173764, 2020.

[24] X. Liu and Y. Liu, "Fractional differential equations with fractional non-separted boundary conditions," Electronic Journal of Differential Equations, vol. 25, p. 1, 2013.

[25] H. H. Alsulami, S. K. Ntouyas, R. P. Agarwal, B. Ahmad, and A. Alsaedi, "A study of fractional-order coupled systems with a new concept of coupled non-separated boundary conditions," Boundary Value Problems, vol. 68, 2017.

[26] S. N. Rao and M. Alesemi, "On a coupled system of fractional differential equations with nonlocal non-separated boundary conditions," Advances in Difference Equations, vol. 2019, no. 1, p. 97, 2019.

[27] Y. Xing, F. Jiao, and F. Liu, "On the generalization of a solution for a class of integro-differential equations with nonseparated integral boundary conditions," Mathematical Problems in Engineering, vol. 2020, Article ID 8679465, 2020.

[28] D. Luo, Ak. Zada, Sh. Shaleena, and M. Ahmad, "Analysis of a coupled system of fractional differential equations with nonseparated boundary conditions," Advances in Difference Equation, vol. 2020, p. 590, 2020. 
[29] A. Krasnoselskii, "Two remarks on the method of successive approximations," Uspekhi Matematicheskikh Nauk, vol. 10, pp. 123-127, 1955.

[30] D. H. Griffel, Applied Functional Analysis, Ellis Horwood, Chichester, UK, 1981. 